\title{
Road Surface Mapping Using Kinect Sensor for Road Maintenance
}

\author{
Yuki CHIN ${ }^{\mathrm{a}}$, Takeki OGITSU ${ }^{\mathrm{b}, *}$, Hiroshi MIZOGUCHI ${ }^{\mathrm{a}}$ \\ aTokyo Unicersity of Science, Yamazaki2641 Noda City, Chiba 278-8510, Japan \\ ${ }^{\text {b} G u n m a ~ U n i v e r s i t y, ~ 1-5-1, ~ T e n j i n-c h o, ~ K i r y u-s h i, ~ G u n m a ~ 376-8515, ~ J a p a n ~}$ \\ *Corresponding Author: ogitsu@ gunma-u.ac.jp
}

\begin{abstract}
Japan currently faces the problem of deterioration of infrastructure such as roads and buildings that were constructed during a period of rapid growth after World War II. Decrepit infrastructure can lead to accidents and disasters. However, maintenance and repair of such infrastructure is expensive. This makes it important to reduce the cost of these operations. In addition, because of vehicle traffic and changes in the environment temperature, roads expand/shrink and crack, leading to potholes. Without repair, potholes can expand gradually and may lead to accidents. To prevent such accidents, it is necessary that roads are inspected and diagnosed to detect cracks and potholes early and repair them. However, currently, road inspection is primarily a visual inspection method, which is time consuming and expensive to perform. If road inspection can be automated using affordable sensors, time and money can be saved. Moreover, vehicles can be equipped with sensors to collect data of the road status, which can be used to create road surface maps that show the location and size of cracks and potholes. This paper focuses on applying a road inspection method using range image sensors to greatly reduce the inspection costs. Specifically, the results of the map of a simulated road with features and the effectiveness of the method are reported.
\end{abstract}

Keywords: Kinect Sensor, Road Surface Map, 3-D Point Cloud

\section{Introduction}

Japan experienced a period of rapid growth in 1955 after World War II, and it continued for 20 years. Various infrastructure such as roads, bridges, and water and gas supply systems were developed during this period. The infrastructure has enabled the Japanese to live in affluence so far. However, as 50 years have passed since the development of the infrastructure, the problem of deterioration of infrastructure has led to accidents and disasters. In 2012, a ceiling slab fell in the Sasago Tunnel in Yamanashi Prefecture, Japan, leading to the death a few people. Such accidents have also occurred outside Japan. For example, in 2006 the bridge collapse in the Mississippi River in Minnesota, USA, 2006 caused many human casualties. These accidents underline that deterioration of infrastructure is a universal problem. Therefore, effective inspection and repair of infrastructure should be conducted to ensure safety and prolong the life of infrastructure.

Potholes and cracks in roads are generated because of expansion/shrinkage of roads by vehicle traffic and temperature variation. Without repair, they can lead to accidents such as vehicle tire blowout and bicycle rollover. Inspection of potholes and cracks to prevent these accidents is primarily a visual inspection method, which is expensive and time consuming.

If roads inspection can be performed automatically by using affordable sensors, a reduction in the required time and money can expected. Moreover, vehicles can be equipped with sensor to collect data of road surface conditions, which can be used to create road surface maps. This paper focuses on applying a road inspection method using range image sensors, which are becoming increasingly more affordable. Specifically, it reports the results of the map of a simulated road with various features and the effectiveness of the method.

\section{Related Works}


The problem of visual inspection of roads by humans is that road inspection tends to be subjective and may overlook abnormal features. Therefore, numerous studies of automatic road inspection have been conducted. For example, one study performed automatic detection of potholes by image processing of the data obtained with a robot-mounted camera $^{(1)}$. Another study performed automatic detection of cracks based on entropy from the images of the road surface obtained with a vehicle-mounted camera ${ }^{(2)}$. In Japan, the inspection car "ROAD TIGER" is used on highways to collect road surface data while moving at a speed $100 \mathrm{~km} / \mathrm{h}$. Road mapping was performed using geo-referenced feature images in a previous study ${ }^{(3)}$.

There have also been studies of road inspection by using image processing techniques, laser sensors, etc. ${ }^{(4,5)}$ However, road inspection by image processing cannot identify the depth of potholes and cracks because the images do not contain sufficient depth information. Moreover, sensors such as laser scanners and inertial measurement units are costly. Given that the depth of potholes and cracks is an important index of the road condition, the cost of road inspection should be reasonable. Therefore, it is necessary to not only detect potholes and cracks reasonably but also measure their depth.

This study aimed to create road surface maps from data of road surface conditions collected by affordable sensors. Existence of potholes and cracks and their size can be determined from the road surface maps. This information can be used to prioritize the sections of roads that require repair.

\section{Creation of Road Surface Map Using Kinect Sensor}

\subsection{Concept}

The crucial aspect of creating a road surface map is alignment of various road surface data. It can be done accurately when data have features such as potholes. However, road surfaces without distinctive features can also exist, which makes it difficult to align their data with the data of other road surfaces. To address this problem, this study scattered objects with features on roads at regular intervals by using a vehicle. Data were collected after scattering the objects, and then the objects were removed by the rear part of the vehicle or by another vehicle. Thus, it was possible to align data of road surfaces with no features. Figure 1 illustrates the concept of this study.
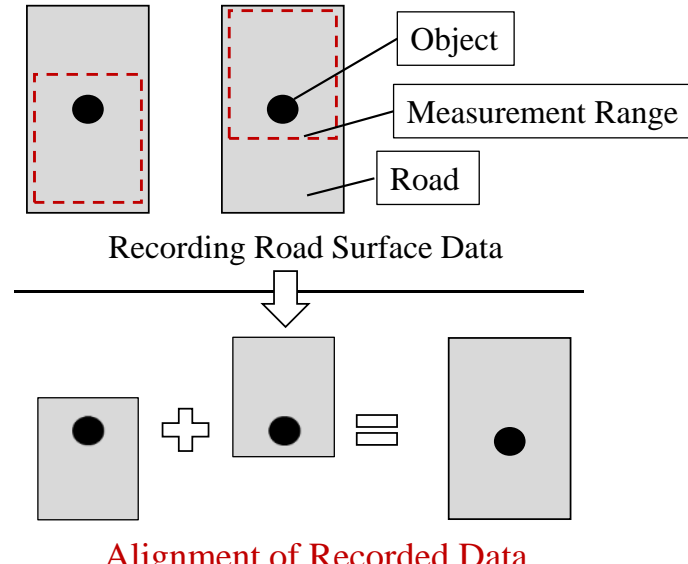

Fig. 1. Concept of This Study.

\subsection{Kinect Sensor}

The Kinect for Windows v2 Sensor (hereinafter referred to as the Kinect sensor (Figure 2) was used as the sensor to collect data. The Kinect sensor is a contactless controller for playing videogames. It consists of a camera and an infrared camera sensor. It detects the position and skeleton of the human by obtaining color and distance information. The Kinect sensor is more affordable than other sensors and therefore presents an opportunity to reduce road inspection costs. The Kinect sensor obtains distance information by the time of flight (TOF) method. In the TOF method, distance is calculated from the time it takes for the irradiated infrared light from the sensor to return to the sensor. The Kinect sensor converts irradiated infrared light to a pulse wave. Therefore, the sensor can be used in open spaces where other sensors are affected by sunlight.

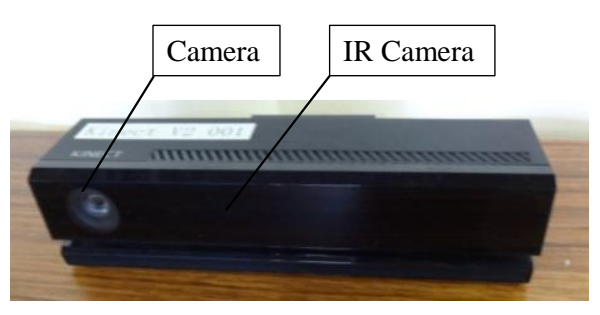

Fig. 2. Kinect for Windows v2 sensor.

\subsection{3-D Point Cloud Information}

A 3-D point cloud is a point cloud in a 3-D rectangular coordinate system such as $(\mathrm{x}, \mathrm{y}, \mathrm{z})$. As the Kinect sensor has an infrared camera sensor and functions according to the TOF method, it can obtain the coordinates of the point of reflection of the infrared light. 
Regarding 3-D point cloud information, open source libraries are available for image processing. Therefore, it is possible to detect surfaces and people. Moreover, 3-D point clouds can be colored using color information obtained by a camera. Therefore, image processing can be performed in colored 3-D point clouds

\subsection{Alignment by Iterative Closest Point Algorithm}

When a road surface map composed of 3-D point cloud information is created, it is necessary to align the point cloud information obtained at regular intervals by unifying the coordinates. In this research, the alignment was performed using the iterative closest point (ICP) algorithm ${ }^{(6)}$. The ICP algorithm minimizes the distance between the corresponding points of two point clouds. It performs rigid body conversion (translation and rotation) to minimize the distance between the corresponding points by searching the nearest points of one point cloud corresponding to those of another point cloud.

\subsection{Downsampling}

Alignment of point cloud data is extremely computation-heavy because the data have an enormous number of points. Therefore, point cloud data were reduced by voxel grid downsampling. A voxel is a regular lattice unit in 3-D space. Voxel grid downsampling replaces all points of a voxel with the center of gravity of the voxel. Thus, the time required for alignment by the ICP algorithm and the data size can be reduced by downsampling.

\section{Experiment}

\subsection{Experimental Conditions}

An experiment was conducted to confirm the allowable size of objects and the amount of movement of the Kinect sensor for aligning point cloud data. The Kinect sensor can collect point cloud data at a rate of $30 \mathrm{fps}$. Therefore, if alignment of point cloud data can be performed successfully when the Kinect sensor is placed at a distance of $0.1 \mathrm{~m}$, then it can be used at a speed $10 \mathrm{~km} / \mathrm{h}$. Figure 3 shows the experimental conditions. The Kinect sensor was set on a wagon and at a distance of $1 \mathrm{~m}$ from the floor. The infrared camera sensor was directed to the floor. After objects were set along the Y-axis, which was the advancing direction of the wagon, point cloud data were obtained. Subsequently, the wagon was moved along the Y-axis and point cloud data were obtained again. In this experiment, five M20 nuts piled

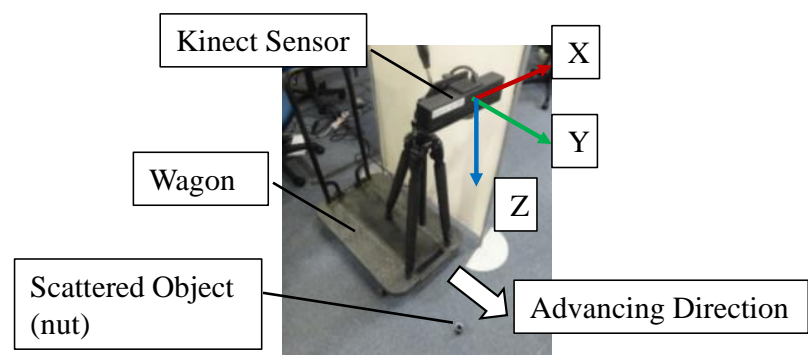

Fig. 3. Conditions of the Experiment.

together were used as the object. Point cloud data are obtained over a length of $300 \mathrm{~mm}$ at $50 \mathrm{~mm}$ intervals. After all point cloud data were obtained, they were reduced by downsampling and data before and after the movement were aligned using the ICP algorithm. Prior to using the ICP algorithm, a transformation matrix was multiplied with one point cloud to align the data in advance. In practice, the point cloud data can be obtained at regular intervals. Thus, it is possible to calculate the transformation matrix to unify the coordinates. In this experiment, the accuracy of the point cloud data alignment, i.e., the extent of overlapping between the data of point clouds of two scattered objects, was estimated.

\subsection{Experiment Result}

Figure 4 shows the distance between two point cloud data of scattered objects. The results confirm accurate alignment even when the Kinect sensor was moved by 100 $\mathrm{mm}$ because the point clouds of scattered objects deviated by a maximum of $20 \mathrm{~mm}$ (Figure 5). However, in the prealigned point cloud data, the point cloud of scattered objects do not overlap with the data obtained after the sensor was

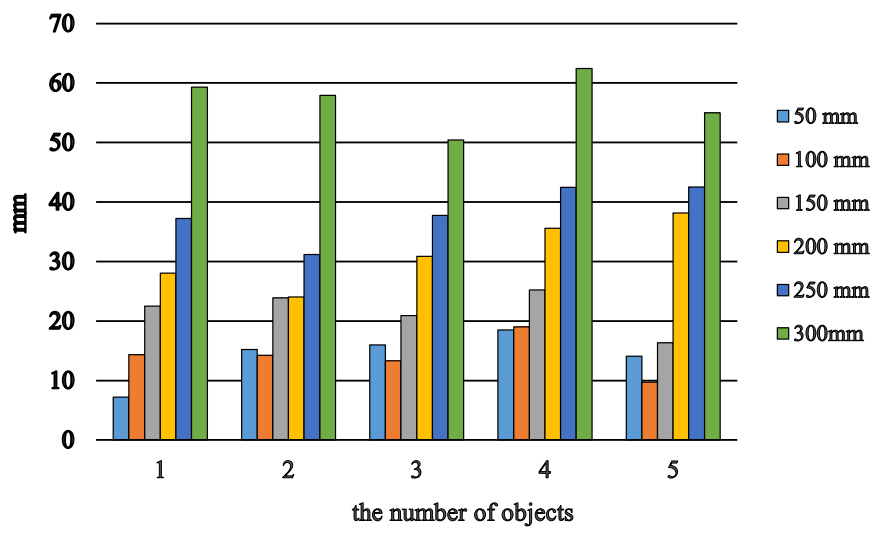

Fig. 4. Distance between Point Clouds of Scattered Objects. 


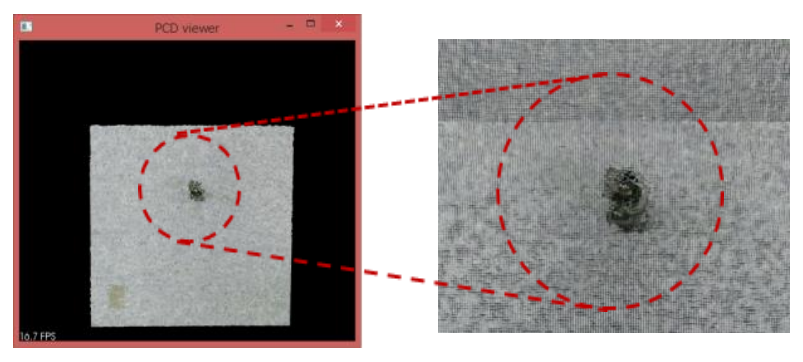

Fig. 5. Example of Successful Alignment.

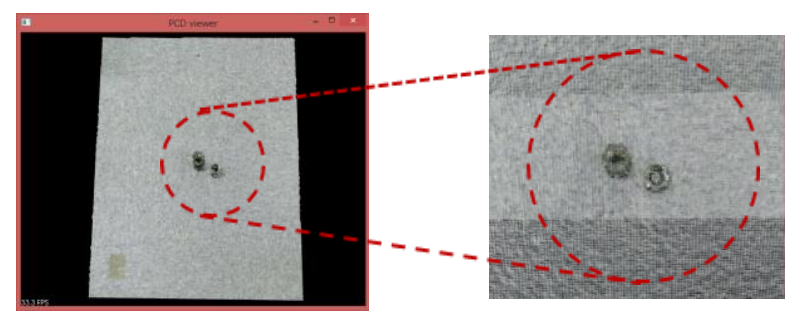

Fig. 6. Example of Failed Alignment.

moved by $100 \mathrm{~mm}$ (Figure 6). This was because the point cloud data after the movement contain the data of the new area that are not available in the point cloud data before the movement. Therefore, it is difficult to accurately align two point cloud data because several point clouds that do not require calculation exist.

On the basis of the results, it can be confirmed that point clouds of a road surface without features can be aligned.

\section{Conclusion}

In this paper, we have described a road surface mapping method using the Kinect sensor for road maintenance and the experiment results obtained using the method. According to the results, even if a road surface does not have features, a road surface map can be created by to setting objects on the road. As future work, topics such as aligning the placement of objects with the sensor and techniques for achieving accurate alignment when the movement of the sensor is beyond $100 \mathrm{~mm}$ will be considered.

\section{References}

(1) Christian Koch and Ioannis Brilakis: "Pothole detection in asphalt pavement images", Advanced Engineering Informatics, Vol. 25, No. 3, pp. 507-515, 2011

(2) Henrique Oliveira and Paulo Lobato Correia:
"Automatic road crack segmentation using entropy and image dynamic thresholding", European Signal Processing Conference (EUSIPCO), pp. 622-626, 2009

(3) Haiyan Guan, Jonathan Li, Yongtao Yu, Michael Chapman, and Cheng Wang: "Automated road information extraction from mobile laser scanning data", Vol. 16, No. 1, pp. 194-205, 2015

(4) Jin Lin and Yuyu Liu: "Potholes detection based on SVM in the pavement distress image", Distributed Computing and Applications to Business Engineering and Science (DCABES), pp. 544-547, 2010

(5) He Youquan, Wang Jian, Qiu Hanxing, Zhang wei, and Xie Jianfang: "A research of pavement potholes detection based on three-dimensional projection transformation", Image and Signal Processing (CISP), Vol. 4, pp. 1805-1808, 2011

(6) Szymon Rusinkiewicz and Marc Levoy: "Efficient variants of the ICP algorithm", Third International Conference on 3D Digital Imaging and Modeling (3DIM), pp. 145-152, 2001 